Márta Fata

\title{
Die deutsche Einwanderung in Ungarn unter Joseph II. Ursachen, Folgen und gesamtmonarchische Zusammenhänge
}

\begin{abstract}
Historical research has not yet adequately answered why there was a large-scale, state-organized settlement of German colonists under Joseph II between 1784 and 1787. Previous studies recognize the only reason for this in the low population density generally characterizing the eastern half of the Habsburg Monarchy. In the present study, the causes and consequences of immigration are placed in overall monarchical contexts and examined. This study concludes that state sponsored immigration of German settlers was no priority of the monarch under the reign of Joseph II. The emperor wanted to reform the contemporary agricultural relations in Hungary too but the strong resistance of the Hungarian nobility resulted that he could not introduce the Raab system in contrast to Czechland and Moravia. The subdivision of state lands directed a part of the German migration to Galicia. The German migration helped him to reach two goals. First, it increased the number of peasant workers; second, a portion of state lands became peasant-owned. The subdivision was only an episode on the way to a new agricultural system. It did not reach the significance of other edicts for instance the abolition of serfdom in 1785. However, its beneficial social and economic effects became clear in the long run.
\end{abstract}

Keywords: Populationismus, Einwanderung, Joseph II., Habsburgermonarchie, Kolonisten

Die historische Forschung hat bislang nicht ausreichend beantwortet, warum es nach der vom Beginn des 18. Jahrhunderts bis in die 1770er-Jahre kontinuierlich erfolgten Einwanderung von Deutschen ab 1784 nochmals zu einer großangelegten, staatlich organisierten Ansiedlung deutscher Kolonisten kam. Die bisherigen Untersuchungen erkennen die einzige Ursache dafür in der die östliche Hälfte der Habsburgermonarchie allgemein charakterisierenden niedrigen Bevölkerungsdichte. Dies ist wenig überraschend, hat doch Joseph II. selbst dieses Argument vorgeführt. Doch die Einwanderung deutscher Kolonisten zwischen 1784 und 1787 muss in einen größeren Zusammenhang gestellt werden, der auch die zeitgenössischen Vorstellungen über den Populationismus und die gesamtmonarchischen Reformbestrebungen des Monarchen in Betracht zieht. ${ }^{1}$

Bekanntlich löste Joseph II. am 12. Januar 1782 nach den deutschen Erblanden auch in den Ländern der ungarischen Krone die kontemplativen Orden auf. Der Besitz der von ihm aufgelösten Orden und des bereits unter Maria Theresia 1777 abgeschafften Jesuitenordens ergab zusammen mit den 504 Kameral- und Krongütern (ohne das Banat) 15 Prozent der urbarialen Grundfläche des ungarischen Königreichs. ${ }^{2}$ Der in staatlicher Hand so angesammelte Grundbesitz warf das Problem seiner effizienten Nutzung auf. Es stellte sich die Frage, ob die Güter verkauft und somit in privatherrschaftliches Eigentum gelangen oder ob sie an die meistbietenden Privatpersonen verpachtet oder eher als bäuerliche Kleinbetriebe parzelliert

\footnotetext{
1 Die folgenden skizzenhaften Ausführungen beruhen auf folgenden Untersuchungen: Fata, Márta: Migration im kameralistischen Staat Josephs II. Theorie und Praxis der Ansiedlungspolitik in Ungarn, Siebenbürgen, Galizien. Münster 2014; Dies.: Migration als Modernisierungsfaktor? Ursachen der deutschen Einwanderung in Ungarn zur Regierungszeit Josephs II. In: Die Ansiedlung der Deutschen in Ungarn. Beiträge zum Neuaufbau des Königreiches nach der Türkenzeit. Hg. v. Seewann, Gerhard - Krauss, Karl-Peter - Spannenberger, Norbert. München 2010, 61-79.

2 Vgl. dazu u. a. Fónagy, Zoltán: Nemesi birtokviszonyok az úrbérrendezés korában. In: Századok 133 (1999), 1167.
} 
werden sollen. Gegen den Verkauf der Staatsgüter an Privatpersonen sprach die Erfahrung mit der Privatisierung der Banater Staatsdomäne. Nicht nur ging der Verkauf eher verschleppend voran, aber bei der Reinkorporierung des Temeswarer Banats in das Königreich Ungarn im Jahre 1778 wurde dort die von den ungarischen Rechtsverhältnissen abweichende Stellung der bäuerlichen Untertanen als Eigentümer ihrer Höfe garantiert. ${ }^{3}$ Sie hatten das Recht, sich von den Frondienstleistungen gegen Entgelt zu befreien. Dennoch erreichte die Zentralorgane eine Flut von Beschwerden gegen die neuen Grundbesitzer vor allem wegen der abverlangten Robotleistungen. ${ }^{4}$

Auch aufgrund dieser Erfahrungen entschied sich Joseph II., der seit seiner Jugend für die Einschränkung der adeligen Vorrechte war, gegen den Verkauf der Staatsgüter. Eine Lösung für die Reformierung der Agrarverfassung erkannte er in der sogenannten Raabisierung. In Böhmen und Mähren begann man zur Regierungszeit Maria Theresias Staatsgüter zu parzellieren und unter den Bauern zu verteilen, die somit nach ihrer Rechtsstellung zu Besitzern ihrer Höfe wurden. Dieses durch den Direktor der böhmischen Kameralgüter, Franz Anton Raab, 1774 entwickelte System ${ }^{5}$ brachte in kurzer Zeit bedeutsame Ergebnisse: Auf den böhmischen und mährischen Kameralgütern, wo zwischen 1767 und 1776 im Jahresdurchschnitt noch 184.000 Gulden in die Staatskasse geflossen waren, stiegen die Einnahmen nach der Parzellierung im Jahre 1778 auf 300.000 und 1781 sogar auf 431.000 Gulden an. ${ }^{6}$ Ein weiterer Erfolg des neuen Systems war die Entladung sozialer Spannungen in den Dörfern. Denn bereits in den ersten zwei Jahren meldeten sich etwa 5.800 Personen, darunter zahlreiche Kleinhäusler und vom väterlichen Erbe ausgeschlossene Bauernsöhne an, um einen Hof zu erhalten. In Böhmen wurden 128 neue Dörfer mit 7.820 Bauernhöfen und in Mähren 117 neue Gemeinden mit 3.628 Höfen errichtet. ${ }^{7}$ Das System bewirkte ein Wachstum, das in der Erweiterung der kultivierten Ackerflächen und in der Zunahme der Agrarproduktion zum Vorschein kam.

Angesichts dieser Erfolge gab Joseph seine noch als Mitregent geäußerte Meinung auf, wonach allein die Großbetriebe einen nennenswerten Gewinn für den Staat abgeben würden. Gleichzeitig hegte er die nicht unbegründete Hoffnung, dass die Einführung des Raabschen Systems eine allgemeine Reformierung der bestehenden Verhältnisse auf dem Land anstoßen würde. Sein am 1. November 1781 erlassenes Patent für Böhmen, Mähren und Schlesien, das die Aufhebung der Leibeigenschaft verfügte, sollte die Rechtsgrundlage für die Reformierung der Agrarverfassung sichern. Das 1783 länderweise erlassene Patent über die Robotabolition ordnete wiederum die Ablösung der Frondienstleistungen durch Geld- und Naturalzahlungen an. ${ }^{8}$ Die Raabisierung und die Patente zielten auf die Etablierung einer von allen grundherrschaftlichen Lasten entbundene freie Bauernschaft mit eigenen Höfen ab. Allerdings konnten die Patente zunächst nur in den österreichischen-böhmischen Ländern durchgeführt werden.

Die Pläne des Monarchen, mit der Raabisierung und der Robotabolition auch in Ungarn die Grundlagen einer neuen Agrarverfassung zu legen und die Staatsdomänen in bäuerliches

\footnotetext{
3 Die Bauern im Banat galten - anders als die in im Königreich Ungarn - nicht als Leibeigene. In den Jahren zwischen 1768 und 1779 wurden ihre Höfe und Ackerfelder ausgemessen und diese ihnen übertragen. Somit hatten sie das Recht, ihre Höfe frei weiterzuvererben und sogar zu veräußern. Zu den Unterschieden vgl. u. a. Mailáth, Johann: Das ungrische Urbarialsystem oder des Grundherrn und des Bauers Wechselverhältnis in Ungern. Pest-Leipzig 1838, 74f.

4 Hegedüs, Antal: Bánáti új földesurak és jobbágyaik 1782-ben. In: Létünk 2 (1978), 108-123.

5 Raab, Franz Anton: Unterricht über die Verwandlung der kais. königl. böhmischen Domainen in Bauerngüter. Wien 1777, 46-48.

6 Vgl.zu den Angaben Barta, János: A felvilágosult abszolutizmus agrárpolitikája a Habsburg- és a HohenzollernMonarchiában. Budapest 1982, 134.

7 Handbuch der Geschichte der böhmischen Länder. Hg. v. Bosl., Karl. Stuttgart 1974, Bd. 2, 491.

8 Lütge, Friedrich: Die Robot-Abolition unter Kaiser Joseph II. In: Wege und Forschungen der Agrargeschichte. Festschrift für Günther Franz. Hg. v. Heinz Haushofer - Willi A. Boelcke. Frankfurt a. M. 1967, 153-170.
} 
Eigentum zu überführen, stießen dort auf starken Widerstand nicht nur der Grundbesitzer, sondern auch der Landesverwaltung. Es begann ein langes Tauziehen zwischen dem Monarchen und der Ungarisch-Siebenbürgischen Hofkanzlei als Vertreterin der ungarischen Interessen in Wien. Das Ergebnis war, dass die Parzellierung von Kameral- und Krongütern im Königreich Ungarn mithilfe der deutschen Siedlungsmigration begann und zum Großteil auch erfolgte, die von Joseph II. ursprünglich jedoch nicht vorgesehen war. Die Siedlungsmigration gehörte zwar in Ungarn schon seit Anfang des 18. Jahrhunderts zu einem der wichtigsten Vehikel der ökonomischen Entwicklung im Land, die bis Ende der maria-theresianischen Zeit sowohl vom Staat als auch von den Grundbesitzern gleichermaßen praktiziert wurde, aber die Einwanderung aus dem Ausland benötigte am Anfang der 1780er-Jahre weder staatlich noch grundherrschaftlich organisierte Rahmen mehr.

Anders gestaltete sich die Lage in dem von der Habsburgermonarchie erst 1772 okkupierten Galizien. Das südwestliche Gebiet Polen-Litauens galt in Wien ökonomisch als besonders unterentwickelt, weshalb sich Maria Theresia eine Anwerbung von Gewerbebetreibenden und Handwerkern gestattete. Das Ziel war jedoch weder eine zahlenmäßig größere Einwanderung noch die Ansiedlung bäuerlicher Kolonisten aus dem Ausland. ${ }^{9}$ Auch Joseph II. bezweckte nach seiner Thronbesteigung 1780 nicht die Erweiterung der Bevölkerungszahl durch Ansiedlung von fremden Einwanderern, sondern die Verbesserung der Lebensverhältnisse der galizischen Bauern. Joseph Brigido, Gouverneur Galiziens, schlug jedoch in der Sitzung des Staatsrates am 18. März 1781 vor, nicht nur für die Einwanderung von nach wie vor fehlenden Handwerkern Anreize zu schaffen, sondern auch deutsche Musterbauern in den zahlreich vorhandenen Kameralherrschaften und Krongütern anzusiedeln. ${ }^{10}$ Brigido, der nach der Leitung der Landesadministration im Banat zwischen 1774 und 1778 den Posten des Vize-Gouverneurs, dann ab 1780 des Gouverneurs in Galizien bekleidete, hoffte aufgrund seiner im Banat gesammelten Erfahrungen, dass die deutschen Kolonisten zum Vorbild für die galizischen Bauern in der Bewirtschaftung ihrer Höfe werden könnten. Das daraufhin am 17. September 1781 erlassene Einwanderungspatent Josephs II. für Galizien bezweckte somit nicht eine Masseneinwanderung. ${ }^{11}$ Die wenigen deutschen Einwanderer sollten auch nicht im römisch-deutschen Reich, sondern in dem von Preußen okkupierten polnischen Gebiet angeworben werden, um so zugleich den preußischen Rivalen zu schwächen. Die in kleinem Umfang begonnene Ansiedlung nahm jedoch bald eine immer größere Dimension an, nachdem 1782 ein übereifriger Kameraldirektor anscheinend auf eigener Faust die Idee hatte, mit dem kaiserlichen Patent um bäuerliche Ansiedler im Rheingebiet zu werben.

Die Werbung löste im römisch-deutschen Reich einen so lebhaften und zugleich anhaltenden Widerhall aus, sodass der Monarch angesichts der rasch wachsenden Einwandererzahl die Provinzverwaltung mahnen musste: Er erinnerte daran, dass das Hauptziel der Reformen auf dem Land und somit der Zweck der Einwanderung, die Förderung der eigenen Untertanen seien. Die eigenen fleißigen und gut wirtschaftenden Untertanen und ihre Söhne sollen an der Parzellierung der Staatsgüter teilnehmen und dadurch in den Stand versetzt werden, eigene Höfe einzurichten. Sie dürfen auch nicht zugunsten der deutschen Einwanderer benachteiligt werden und sollten die gleichen Mittel und Hilfe bekommen, wie die deutschen Siedler. ${ }^{12}$

\footnotetext{
9 Rosdolsky, Roman: Untertan und Staat in Galizien. Die Reformen unter Maria Theresia und Joseph II. Mainz 1992. 10 Österreichisches Staatsarchiv [ÖStA] Haus-Hof- und Staatsarchiv [HHStA], Kabinettsarchiv [KA], Staatsratprotokolle 1781: 2055. Vgl. auch Rumpel, Hubert: Die Reisen Kaiser Josephs II. nach Galizien. Diss. Erlangen 1946, 143.

11 ÖStA Finanz- und Hofkammerarchiv [FHKA], Neue Hofkammerarchiv [NHK] Galizische Domänen [Dom Gal], rote Nr. 67, fol. 200r-207v.

12 ÖStA HHStA, KA, Staatsratprotokolle 1782: 2027. Vgl. auch Rumpel, Die Reisen, 161, Fußnote 7.
} 
Das bescheidene Ergebnis des 1783 in zwei Kameralherrschaften probeweise eingeführten Systems verfestigte allerdings sowohl im galizischen Gubernium als auch in der BöhmischÖsterreichischen Hofkanzlei als Oberbehörde der Provinz in Wien, die Ansicht, dass die Raabisierung und die Robotabolition nur im Fall einer kontinuierlichen Marktproduktion möglich sei. ${ }^{13}$ Nach einstimmiger Meinung fehlten jedoch die Bedingungen dafür in Galizien, und dem galizischen Bauer mangelte es an Kenntnissen und Mut, um seine traditionelle Produktionsweise zu verändern und sich auf die Marktproduktion umzustellen. Vorgeschlagen wurde deshalb die Ansiedlung von Reichsdeutschen in allen galizischen Kreisen, die den Einheimischen mit gutem Beispiel vorangehen sollten. ${ }^{14}$ Joseph II. genehmigte zwar die Fortsetzung der deutschen Einwanderung, bestand jedoch weiterhin auch auf die Parzellierung von Grundstücken unter den einheimischen Bauern. Dieses Ziel rückte allerdings wegen der schnell zunehmenden Zahl an Einwanderern immer mehr in den Hintergrund. Bis 1787, als die deutsche Siedlungsmigration ganz eingestellt wurde, wurden 13.000 deutsche Einwanderer und lediglich einige hundert galizische Bauern angesiedelt. ${ }^{15}$

Die ungarischen Behörden mussten sich gezwungenermaßen mit den galizischen Angelegenheiten auseinandersetzen, als sich Joseph II. 1784 entschloss, die Siedlungsmigration wegen des auf Galizien lastenden Drucks der Einwandererzahlen nach Ungarn umzulenken. Er musste bis dahin mit der ungarischen Landesverwaltung, die die Einführung eines neuen Systems im Königreich Ungarn ablehnte und in ihren Denkschriften an den Herrscher ganz ähnlich wie der Staatsrat und die galizischen Verwaltungsorgane argumentierte, zäh verhandeln. ${ }^{16}$ Die Ungarisch-Siebenbürgische Hofkanzlei bemühte sich, den Monarchen von der Unausführbarkeit seines Vorhabens auch schon deshalb zu überzeugen, weil sie durch die stufenweise Modifizierung der Agrarverfassung letztendlich die Abschaffung der adeligen Privilegien befürchtete. Grund für diese Annahme lieferte etwa die von Joseph am 31. März 1783 erteilte Antwort, dass ihn keineswegs nur fiskalische Gesichtspunkte wie etwa die Erhöhung des Staatseinkommens leite, sondern überhaupt die Einführung eines neuen Wirtschaftssystems, das u. a. die Abschaffung des Rechts, wonach nur Adelige Grund und Boden besitzen dürfen, ins Visier nehme. Joseph II., der entschlossen war, seine Pläne auch in Ungarn durchzuführen, erkannte in der deutschen Siedlungsmigration eine willkommene Gelegenheit, wenigstens die Sache der Parzellierung in Gang zu bringen. ${ }^{17}$

Im Gegensatzzu Galizien wies Joseph II.im Fall Ungarns an erster Stelle nicht auf gravierende gesellschaftliche und soziale Probleme hin, sondern auf die Tatsache, dass sich das Land nicht in dem Maße entwickele, was seine ansonsten guten natürlichen Voraussetzungen durchaus ermöglichen könnten. ${ }^{18}$ Eine der Hauptursachen erkannte er in der niedrigen Bevölkerungszahl. Die Agrarreformen in Ungarn setzten seiner Ansicht nach mehr als anderswo in der Monarchie ein Bevölkerungswachstum voraus. Mit dieser Ansicht war er keineswegs allein. Unter den Wirtschaftsautoren der Zeit hob auch Sámuel Tessedik in seinem 1784 erschienenen Werk „Der Landmann in Ungarn, was er ist und was er sein könnte“ die Tatsache hervor, dass der ungarische Bauer mit dem Ackerboden immer noch verschwenderisch umgeht und die längst veraltete Zweifeldwirtschaft verwendet. Die Ursachen dafür erkannte Tessedik nicht zuletzt

\footnotetext{
13 ÖStA FHKA, NHK, Dom Gal, rote Nr. 67, fol. 297r.

14 Ebd., rote Nr. 71, fol. 1163v-1164r.; Kaindl, Raimund Friedrich: Geschichte der Deutschen in den Karpathenländern. Bd. 3. Gotha 1911, 30.

15 Ebd., rote Nr. 74, fol. 560r-563v.

16 Magyar Nemzeti Levéltár [MNL] Országos Levéltár [OL] A 39: Generalia 1784/335.

17 ÖStA HHStA KA, Staatsratprotokolle 1783/1209; MNL OL A 39: Generalia, 1783/3185, Pos. 1.

18 Vgl. dazu Szántay, Antal: Regionalpolitik im alten Europa. Die Verwaltungsreformen Josephs II. in Ungarn, in der Lombardei und in den österreichischen Niederlanden 1785-1790. Budapest 2005, 62.
} 
in der stellenweise niedrigen Bevölkerungszahl, in deren Folge noch große Gebiete in den Dorfgemarkungen und zwischen den Dörfern der Ungarischen Tiefebene ganz brachliegen. ${ }^{19}$

Als im Jahre 1783 die Zahl der deutschen Einwanderer in Galizien sprunghaft zunahm, sodass ihre Ansiedlung stockte, mussten sie für lange Monate auf Staatskosten in provisorischen Unterkünften untergebracht und versorgt werden. Die Beamten der Böhmisch-Österreichischen Hofkanzlei befürworteten nichtsdestotrotz die Fortführung der Einwanderung. Denn sie waren nicht nur von den populationistischen Ideen des Kameralismus geleitet, sondern auch von der Befürchtung, dass die Einstellung der Siedlungsmigration die Einwanderer nach Preußen oder Russland führen könnte, die ebenfalls um Einwanderer warben. Die Bevölkerungsvermehrung war in der östlichen Hälfte Europas im letzten Drittel des 18. Jahrhunderts nach wie vor eine wichtige Staatsaufgabe, ${ }^{20}$ basierte doch die wirtschaftliche und militärische Kraft der Monarchie auf der Zahl der steuerzahlenden und Soldaten stellenden Untertanen. Die Böhmisch-Österreichische Hofkanzlei schlug deshalb vor, die Auswanderungswilligen aus dem Reich nicht zurückzuweisen, sondern sie als Kolonisten in das Königreich Ungarn umzuleiten. Joseph II., selbst ein Befürworter der optimistischen Variante des Populationismus, genehmigte diese Lösung und hielt die Ungarische Hofkammer an, die in Galizien angewandten Methoden, insbesondere die die Kolonisten äußerst begünstigenden Beihilfen und Privilegien zu übernehmen. ${ }^{21}$ Die Übernahme dieser Ansiedlungsbedingungen war schon deshalb unerlässlich, weil die deutschen Einwanderer ihr Umleiten nach Ungarn anfangs ablehnten. Unter den Auswanderern im römisch-deutschen Reich verbreitete sich seit der letzten großen Ansiedlungswelle im Banat unter Maria Theresia der Ruf Ungarns als „Friedhof der Deutschen“. Schuld daran trug die hohe Sterblichkeitsrate unter den Ansiedlern. ${ }^{22}$

Nach der Vorstellung des Monarchen wären die Ansiedlung deutscher Einwanderer und die Raabisierung unter den einheimischen Untertanen parallel und keineswegs zu Lasten der anderen Gruppe durchzuführen gewesen. Allerdings stießen die Pläne nicht nur bei den Landesbehörden, sondern auch in den meisten Kameralherrschaften auf Skepsis und Ablehnung. Nur in der Batschka wurden die Pläne bereits im Mai 1784 in die Planung aufgenommen. ${ }^{23}$ Danach sollten in der Zomborer Kameraladministration deutsche Einwanderer auf öden und bis dahin nicht bebauten Terrains angesiedelt werden, während einheimische Untertanen durch Tod oder Flucht vakant gewordene Bauernstellen und sogenannte Überlandfelder, d. h. von den Gemeinden bis dahin nicht verteilte Felder erhalten sollten. Die Kameraladministration argumentierte sogar für die Ansiedlung einheimischer Kleinhäusler und Bauernsöhne damit, dass diese nicht nur wesentlich weniger Kosten verursachen würde als die der deutschen Einwanderer, weil die Einheimischen weder Reisegelder noch besondere Ausgaben benötigten. Und weil sie auch nur drei steuerfreie Jahre erhalten, könnten sie mit der Kontributionszahlung früher beginnen als die Einwanderer. Der freiwillige Loskauf von der Leibeigenschaft und die Robotabolition wurden nicht in die Pläne eingebaut, aber in den Kameralherrschaften der Batschka praktizierte man schon vor 1784 gelegentlich die Ablösung der Frondienste.

Auch in immer mehr Kameraladministrationen erkannten Beamte -, die gegenüber der Ungarisch-Siebenbürgischen Hofkanzlei sich mit den örtlichen Verhältnissen tagtäglich

\footnotetext{
19 Teschedik, Samuel: Der Landmann in Ungarn, was er ist und was er sein könnte; nebst einem Plane von einem regulirten Dorfe. Pest 1784.

20 Vgl. u. a. Fubrmann, Martin: Volksvermehrung als Staatsaufgabe? Bevölkerungs- und Ehepolitik in der deutschen politischen und ökonomischen Theorie des 18. und 19. Jahrhunderts. Paderborn et al. 2002.

21 Fata, Márta Migration im kameralistischen Staat, 240-246.

22 ÖStA FHKA, NHK, Dom Gal, rote Nr. 68, fol. 361v.; Fata, Márta: „Kirchhof“ versus „Paradies“. Die Auswanderung in Selbstzeugnissen der Kolonisten zur Regierungszeit Josephs II. In: Migration im Gedächtnis. Auswanderung und Ansiedlung im 18. Jahrhundert in der Identitätsbildung der Donauschwaben. Hg. v. Ders. Stuttgart 2013, 39-51.
}

23 MNL OL A 39, 1784/5983. 
auseinandersetzen mussten - in der Parzellierung eine Möglichkeit, der stellenweise zuspitzenden sozialen Lage auf dem Land Abhilfe leisten zu können. ${ }^{24}$ Insbesondere in den dichtbevölkerten oberungarischen Komitaten wurden deshalb die örtlichen Bewohner bevorzugt. Im Komitat Nyitra beispielsweise wurden auf das Prädium Kolos im Mai 1784 nicht die dorthin verwiesenen 31 deutschen Einwanderer, sondern slowakische Bewerber aus der Umgebung angesiedelt. Wegen der sich zunehmende Proteste der Bauern gegen die deutschen Ansiedler erhob auch die sich erst formierende ungarische Öffentlichkeit gegen die deutschen Ansiedler ihr Wort. Die sonst mit dem Wiener Regierung sympathisierende „Preßburger Zeitung“ machte zum Beispiel in ihrem Artikel vom 6. August 1785 darauf aufmerksam, dass aus dem Komitat Árva jährlich etwa 1.500 Personen gruppenweise in andere Komitate arbeiten gehen, um das tägliche Brot zu verdienen, und dass ca. 2.000 Familien in die weniger dicht besiedelten Komitate im Süden des Landes auswandern würden, um dort eine neue Existenz als Bauern aufzubauen. ${ }^{25}$ Auch der Josephinist Graf Antal Jankovics, dem aufgrund seiner zwischen 1783 und 1785 durchgeführten Untersuchung des Räuberunwesens im Banat die dortigen Verhältnisse wohl bekannt waren, schlug dem Herrscher vor, Kleinhäusler, Tagelöhner und Bauernsöhne aus Oberungarn im Banat anzusiedeln. Doch nach Ansicht des Monarchen sollten Komitatsgrenzen überschreitenden Umsiedlungen nur für den Fall vorgesehen sein, dass Reichskolonisten nicht in ausreichender Zahl vorhanden wären. ${ }^{26}$ Die Komitatsgrenzen überschreitende Binnenmigration wurde auch von den Komitaten abgelehnt. Im Fall der Abnahme der steuerzahlenden Bauern hätte man nämlich bei gleichbleibender Bemessung der Kontribution den auf die einzelnen Bauern errechneten Betrag erhöhen müssen. So kam lediglich eine größere grenzüberschreitende Binnenmigration 1785 zustande, als mit Genehmigung Josephs II. 211 Familien aus Kunhegyes in die Batschka umsiedeln und ihnen weitere kleinere Gruppen ebenfalls aus dem freien Distrikt der Jazygen und Kumanen folgen durften. ${ }^{27}$

Der Herrscher, der die Ansiedlung von Deutschen und von Inwohnern zunächst parallel anlaufen ließ, ordnete die Ansiedlung von Binnenmigranten am 10. Oktober 1785 unter dem Druck aus den Kameraladministrationen sogar an, ${ }^{28}$ stellte sie jedoch schon mit der Anordnung vom 10. April 1786 wieder ein. ${ }^{29}$ Seinen Standpunkt begründete er mit der dringend erforderlichen Unterbringung der in Ungarn auf Staatskosten eingetroffenen deutschen Siedler. Hier zeigten sich wieder einmal die Ambivalenzen der josephinischen Reformpolitik.

Bis 1787 wurden in den ungarischen Kameralgütern und in einigen südungarischen Städten über 10.000 deutsche Familien mit mehr als 45.000 Personen angesiedelt. ${ }^{30} \mathrm{Im}$ Gegensatz dazu betrug die Zahl der Binnensiedler zwischen 1.500 und 2.000 Familien, wobei ihre genaue Zahl bisher nicht ermittelt werden konnte. Deutsche Einwanderer wurden hauptsächlich in der Batschka (etwa 3.300 Familien) und im Banat (um 3.000 Familien) angesiedelt. In der Batschka wurden für sie 7, im Banat 14 neue Dörfer gebaut, zudem wurden in beiden Kameralgebieten 23 bereits bestehende Dörfer mit einem neuen Dorfteil für sie erweitert. In den anderen

\footnotetext{
${ }_{24}$ Vgl. dazu Fata, Márta: „Nachdem die Vermehrung der Population [...] der Haupt-Endzweck ist“. Deutsche Einwanderung zur Zeit Josephs II. am Beispiel des Lechnitzer Religionsfonds in der Zips. In: Deutsche Sprache und Kultur in der Zips. Hg. v. Wynfried Kriegleder - Andrea Seidler - Jozef Tancer. Bremen 2007, 123-136.

25 Preßburger Zeitung vom 6. August 1785

26 ÖStA FHKA NHK, Hofresolutionsbücher, Hungarische Resolutionsbücher Bd. 17, fol. 210

27 Szabó, Lajos: Kunhegyesi „földtelen emberek Feketitsre“ költözése 1785-ben. In: Jubileumi tudományos ülés a jászkunságiak bácskai kitelepülésének 200. évfordulóján. Hg. v. Gyöngyi Kaposvári - Gábor Bagi. Szolnok 1989, $37-65$.

28 ÖStA FHKA NHK, Hofresolutionsbücher, Hungarische Resolutionsbücher Bd. 16, fol. 334.

29 Ebd., Bd. 17, fol. 277, 301.

30 Vgl. u. a. Kollega Tarsoly, István: II. József német telepesei 1784-1787. Budapest 2011. https://btk.ppke.hu/ uploads/articles/56720/file/disszertaciok/kollega tarsoly 2012 d k.pdf(31.12.2020).
} 
Kameralbezirken wurden insgesamt mehr als 4.200 neue Höfe für Ansiedler eingerichtet. ${ }^{31}$ Wo es zwischen den deutschen Einwanderern und den einheimischen Untertanen eine Konkurrenz um die Felder gab, kam es gelegentlich auch zu handgreiflichen Zusammenstößen. Doch insgesamt verlief die deutsche Siedlungsmigration reibungslos und wirkte zusammen mit der Binnenmigration positiv auf die Agrarwirtschaft aus: Wurden nämlich 1780 etwa 4 Millionen Joch Acker im Land bebaut, so erhöhte sich die Zahl bis 1786 um 700.000 Joch. ${ }^{32}$

Der neue Untertan war allerdings nicht immer der vom Herrscher und der Kameraladministration erwartete Musterbauer. Laut der Einwandererlisten waren nur 50 Prozent der deutschen Einwanderer tatsächlich Ackerbauer, 30 Prozent machten die Handwerker aus, und 20 Prozent unter ihnen lebten zu Hause gleichzeitig von der Landwirtschaft und einem Handwerk. ${ }^{33}$ Die Einwanderer mit mangelhaften Kenntnissen im Ackerbau bereiteten daher zusätzliche Probleme für die Kammerinspektoren. Im Zomborer Amt notierte Mihály Ürményi, dass die Deutschen in Cservenka, Torzsa und Új-Verbász den Ackerbau nicht beherrschen. Sie können auch ihre Pferde nicht einspannen, und die Schweine halten sie sogar im Haus und den Dünger schaufeln sie einfach durch das Fenster neben die Hauswand, sodass die aus Lehm gebauten Hauswände durchfeuchtet werden. Ürményi ließ deshalb - ähnlich wie die anderen Inspektoren - einheimische Bauern beschäftigen, die die Einwanderer mit den örtlichen Verhältnissen und den erforderlichen Wirtschaftsmethoden vertraut machten. Trotz der Schwierigkeiten wollten die meisten Einwanderer nicht als Gewerbebetreibende oder Tagelöhner tätig werden, sondern als Vollbauern eine Existenz in Ungarn aufbauen. Der Grund dafür ist nicht zuletzt in den für Bauern vorgesehenen zahlreichen Begünstigungen zu suchen, die vom Schriftsteller Mór Jókai aus der Distanz von etwa hundert Jahren etwas ironisch, aber der Wahrheit entsprechend beschrieben wurde: Die Einwanderer haben auf Staatskosten nicht nur fertige und eingerichtete Häuser sowie Zugvieh und Arbeitsgeräte erhalten, sondern sogar die in den Stuben vor dem Ofen schnurrenden Katzen. ${ }^{34}$

Nach Ablauf der den Deutschen eingeräumten zehn steuerfreien Jahre konnten sich die Ansiedler als Bauern auf eigene Füße stellen, wie Johann Eimann, ein 1785 aus Baden-Durlach in die Batschka eingewanderte Mennonit in seinem Werk beschrieb. ${ }^{35}$ Den Fleiß, den Eimann als Willen der Kolonisten interpretierte, beschrieben auch andere Beobachter, darunter der Grundbesitzer und Dichter Dániel Berzsenyi. Er notierte auch einen der Gründe für den Erfolg der Kolonisten, nämlich die Arbeitsorganisation, die - anders als bei den anderen in Ungarn lebenden Völkern - nicht nach Geschlechtern erfolgte. So haben bei den Deutschen auch Frauen und Töchter schwere Feldarbeit verrichtet und Männer sich im Winter am Webstuhl beschäftigt, während der ungarische Bauer vor seinem Haus saß und seine Pfeife rauchte. ${ }^{36}$

Die Nachfolger der anfangs noch mit allerlei Schwierigkeiten kämpfenden Siedler etwa in der Batschka bauten in den 1830er-Jahren Weizen in großer Menge an, und mehrere von ihnen bereicherten sich durch den Getreidehandel. In der ersten Hälfte des 19. Jahrhunderts begann deshalb dort ein Verbürgerlichungsprozess, der sich nach der Bauernbefreiung im Jahre 1848 in der Gründung von Schulen, Zeitungen, Vereinen und Sparkassen zeigte. Die Abkömmlinge der deutschen Einwanderer strebten sich danach, möglichst viele Ackerfelder zu

31 Angaben bei Czoernig, Karl Freiherr: Ethnographie der oesterreichischen Monarchie. Bd. 3. Wien 1858, 66-72.

32 Vgl. die Angaben bei Benda, Gyula: Statisztikai adatok a magyar mezőgazdaság történetéhez 1767-1867.

Budapest 1973, 153, 157.

33 Kollega Tarsoly, II. József, 51-58.

34 Es sei angemerkt, dass Katzen in den Kolonistendörfern in der Tat ausgeteilt wurden, um die rasche Vermehrung der Weizen und Kleider gleichermaßen beschädigenden Nagetiere zu verhindern.

35 Eimann, Johann: Der Deutsche Kolonist, oder die deutsche Ansiedlung unter Kaiser Joseph dem Zweyten in den Jahren 1783-1787 absonderlich im Königreich Ungarn in dem Bácser Comitat. Pesth 1822.

36 Berzsenyi, Dániel: A magyarországi mezei szorgalom némely akadályairól, 1833. In: Berzsenyi Dániel összes mūvei. Hg. v. Oszkár Merényi. Budapest 1968, 329-357. 
erwerben, was schon vor 1848 zu einer zunehmenden Binnenmigration unter ihnen führte. Mit ihrer ökonomischen Expansion ging auch eine religiöse einher, was sich bei den Protestanten besonders anschaulich zum Ausdruck kam. Infolge des josephinischen Toleranzpatentes von 1781 durften auch protestantische Siedler einwandern und ihre Konfession ausüben. In der Batschka entstanden sieben evangelisch-lutherische und evangelisch-reformierte Gemeinden, die infolge der verstärkten Binnenmigration bald eine Reihe von Sekundär- und Tertiärsiedlungen und Kirchengemeinden mit Filialen gründeten. Die Dörfer standen nicht nur zueinander in engen familiären und geschäftlichen Beziehungen, sondern sie knüpften auch $\mathrm{zu}$ anderen protestantischen Gemeinden in Ungarn rege Kontakte. ${ }^{37}$

Zum Schluss soll festgehalten werden, dass die unter Joseph II. auf Staatskosten erfolgte Einwanderung deutscher Ansiedler ursprünglich nicht zur Hauptzielsetzung des Herrschers gehörte. Joseph, der die bestehenden Verhältnisse zwar auch in Ungarn reformieren wollte, konnte die Raabisation und die Robotabolition - anders als etwa in Böhmen oder Mähren wegen des starken Widerstandes des ungarischen Adels nicht durchsetzen. Um dennoch die als notwendig erachtete Parzellierung von Staatsgütern in Angriff zu nehmen, nutzte er die sich durch die deutsche Einwanderung nach Galizien darbietende Gelegenheit. Mithilfe der deutschen Siedlungsmigration konnte er zwei Ziele erreichen: die Vermehrung der Zahl der bäuerlichen Arbeitskräfte und die Überführung von Staatsdomänen in bäuerliches Eigentum. Auf dem Weg zu einer neuen Agrarverfassung war die Parzellierung freilich nur eine Episode und konnte die Bedeutung anderer Verordnungen wie die der Abschaffung der Leibeigenschaft im Jahre 1785 nicht erlangen. Aus der Langzeitperspektive zeigten sich dennoch ihre positiven sozialen und ökonomischen Auswirkungen.

37 Fata, Márta: Wo das Evangelium nicht gehet, da ist keine Kirche. Ursachen, Verlauf und Folgen der Binnenwanderung deutsch-lutherischer Siedler in der Batschka und in Syrmien im 19. Jahrhundert. In: Luther und die Evangelisch-Lutherischen in Ungarn und Siebenbürgen. Augsburgisches Bekenntnis, Bildung, Sprache und Nation vom 16. Jahrhundert bis 1918. Hg. v. Dies. - Anton Schindling. Münster 2017, 141-189. 